\title{
How special is Jupiter?
}

\section{George W. Wetherill}

BUILDING Jupiter has long been a problem to theorists. Observations presented on page 494 of this issue ${ }^{1}$ confirm that the problem is indeed a serious one.

The idea that stars and planetary systems including our Solar System form simultaneously from a disk-shaped nebula of gas and dust is an old one, dating back to the days of Kant and Laplace. In recent decades this hypothesis has finally received considerable observational and theoretical support. Infrared, millimetre and optical wavelength observations of very young stars demonstrate the presence of infalling circumstellar disks of dust and gas associated with these stars. Theoretical calculations have supported plausible mechanisms whereby, in a symbiotic manner, the excess angular momentum associated with a 'core' of a parental interstellar molecular cloud may be partitioned between the newly formed central star and a residual disk of gas and dust. The star thus obtains the mass it needs to function as a hydrogen-burning thermonuclear furnace, while losing the excess angular momentum that would prevent it from collapsing into a compact body. Much of this angular momentum, associated with only a small part of the mass, can be transferred to a residual centrifugally supported cirsumstellar disk from whith planets may form.

At present, our planetary system contains no more than a few per cent of the gas, primarily hydrogen, that should have accompanied a preplanetary disk of cosmic chemical composition. How and when was this excess gas lost? Our well behaved middle-aged Sun is not capable of 'blowing away' such large quantities of gas. It seems most likely that the necessary loss of gas from the plane of the disk would occur during the less stable first few million years of a star's life.

Zuckerman et al. ${ }^{1}$ report radiofrequency observational data supporting this picture for 20 nearby young stars, all estimated to be between 1 and 10 million years of age, using methods of varying reliability. They determined the gas density from the intensity of carbon monoxide molecular transition lines, direct determination of $\mathrm{H}_{2}$ being impossible because of the absence of suitable $\mathrm{H}_{2}$ radiofrequency transitions. Concentrations of $\mathrm{H}_{2}$ were then obtained by assuming the standard interstellar ratio $\left(10^{4}\right)$ between the number of $\mathrm{H}_{2}$ and $\mathrm{CO}$ molecules. Subject to many caveats, the authors conclude that within a few million years after the formation of a star, the mass of $\mathrm{H}_{2}$ gas remaining is typically very small, much less than the mass of Jupiter. An exception to this has been found for the still young star GM Aurigae ${ }^{2}$, which is roughly 2 million years old.

This early loss of gas could make problems for the formation of Jupiter and Saturn. Theoretical models for the formation of Jupiter usually first require the growth of a core roughly 10 times the mass of the Earth, upon which the gas necessary for Jupiter to grow to its full 317 Earth masses will accrete from the disk in a few million years. The requirements for the formation of Saturn are similar. Obviously this entire process must take place before the gas has been lost from the disk. It has proved awkward to develop a quantitative model that permits a sufficiently large Jupiter core to grow during the short time that gas is present, and at the same time is consistent with the absence of large planets in the asteroid belt, although this may be possible for seemingly ad hoc distributions of mass of gas and dust in the disk. An 'easy' way out of this dilemma could be to hypothesize that the special conditions necessary to form Jupiter and Saturn represented an unusual stochastic circumstance, and that planetary systems rarely include gas giant planets, even if their terrestrial planet regions are populated by bodies generally similar to those of our Solar System.

There is some observational support for this. Conventional models for the formation of the 'Oort cloud' of comets, roughly $10^{4}-10^{5}$ astronomical units (AU) distant, predict that this process is inefficient and that many more comets were ejected from the Solar System than were preserved in the Oort cloud. Jupiter (and Saturn) are primarily responsible for this ejection. If star formation were always accompanied by ejection of comets, interstellar space would be well populated by these bodies, which from time to time should penetrate the inner Solar System and be discovered as comets with distinctive hyperbolic orbits. No such comets have been observed, whereas it has been argued that at least one should have been ${ }^{3}$.

More direct evidence for a paucity of extrasolar Jupiter-like planets is suggested by telescopic searches for perturbations of the motion of nearby stars by possible Jupiter-mass companions ${ }^{4-6}$. Although more than 20 stars have been well studied, no evidence has been found for companion planets down to a limit of 1 to 3 Jupiter masses at a distance of $5 \mathrm{AU}$.

If 'Jupiters' are rare, why should we happen to live in an unusual planetary system that contains a Jupiter (and a Saturn as well)? One answer could be that our having a Jupiter is just a random chance. If gas giants can occasionally form somewhere, why not here? A more in- teresting possibility is that if it were not for the gas giant planets, the Earth would not be habitable, at least for advanced organisms that think about questions of this kind. Maybe we must observe a Jupiter in our Solar System because otherwise we wouldn't be here. Instead of producing Jupiters of roughly 300 Earth masses, perhaps lack of synchronization of the independent processes of planetary growth and gas loss would usually lead to hydrogen-poor 'failed' Jupiters of about the mass of Neptune and Uranus.

In such systems the outer Solar System would not be cleansed of residual cometary material, including bodies the size of Pluto or even larger. Instead, most of these bodies could be stored in nearby trans-Neptunian orbits, from whence they could easily penetrate into the inner Solar System over the entire age of the Solar System ${ }^{7}$. Catastrophic impact events of the kind believed to have caused the mass extinctions at the Cretaceous/Tertiary boundary might then have occurred about every $10^{5}$ years instead of about once in $10^{8}$ years. Stabilization of the Earth's obliquity and therefore its climate and habitability by the Moon has been suggested ${ }^{8}$; we may be more dependent on other members of our Solar System than previously thought.

It could justifiably be argued that the theoretically based suggestion that Jupiters are rare is simply an excuse for our ineptitude in producing an adequate theory for the formation of the outer planets. The answer must come from observation. Theory can stitch together threads of observations into a fabric of understanding. The observational data are not yet definitive. Taken altogether, the evidence does raise the possibility that what was thought to be the sine qua non of a 'real' planetary system, the presence of planets like Jupiter and Saturn, may actually be unusual. In contrast, more easily made terrestrial planet systems physically similar to ours may be abundant but hazardous unless protected by gas giant planets.

George W. Wetherill is in the Department of Terrestrial Magnetism, Carnegie Institution of Washington, 5241 Broad Branch Road Northwest, Washington DC 20015, USA.

1. Zuckerman, B., Forveille, T. \& Kastner, J. H. Nature 373 494-496 (1995).

2. Koerner, D. W., Sargent, A. I. \& Beckwith, S. V. W. Icarus 106, 2-10 (1993).

3. Weissman, P. R. in Comets in the Post-Halley Era (eds Newburn, R. L. et al.) 463-498 (Kluwer, Dordrecht. 1994)

4. Walker, G. H. et al. Icarus (in the press)

5. Cochran, W. D. \& Hatzes, A. P. in Planets Around Puisars (eds Philips, J. A. et al.) 267-274 (Astr. Soc. Pac. Conf. Vol. 36,1993 )

6. Marcy, G. W. \& Butler, R. P. Publ. astr. Soc. Pacif. 104 270-277 (1992)

7. Wetherill, G. W. in Planetary Systems: Formation, Evolution and Detection (eds Burke, B., Rahe, J.H. \& Roettger. E. E.) 23-32 (Kluwer, Dordrecht, 1994).

8. Laskar, J. \& Robutel, P. N. Nature 361, 608-615 (1993). 